\title{
GAUGE GRAVITY and ELECTROWEAK THEORY
}

\author{
DAVID HESTENES \\ Physics Department, Arizona State University, \\ Tempe, Arizona 85287-1504, USA \\ hestenes@asu.edu
}

\begin{abstract}
Reformulation of the Dirac equation in terms of the real Spacetime Algebra (STA) reveals hidden geometric structure, including a geometric role for the unit imaginary as generator of rotations in a spacelike plane. The STA and the real Dirac equation play essential roles in a new Gauge Theory Gravity (GTG) version of General Relativity (GR). Besides clarifying the conceptual foundations of GR and facilitating complex computations, GTG opens up new possibilities for a unified gauge theory of gravity and quantum mechanics, including spacetime geometry of electroweak interactions. The Weinberg-Salam model fits perfectly into this geometric framework, and a promising variant that replaces chiral states with Majorana states is formulated to incorporate zitterbewegung in electron states.
\end{abstract}

\section{Introduction}

This conference rightly honors Marcel Grossman as exemplar of the profound influence of mathematics in shaping physical theory. He educated Einstein in the absolute differential calculus of Ricci and Levi-Civita and extended the concept of tensor to covariant systems. As we all know, this calculus provided Einstein with new conceptual structures that were essential for creating his General Theory of Relativity.

The conference parallel session on Geometric Calculus and Gauge Theory Gravity presented a more recent approach to gravity theory where alternative mathematics plays an essential role. Though few physicists are yet conversant with this approach, it is developing vigorously, with a substantial body of new mathematical techniques and physical results. This article is a status report on the field with emphasis on recent developments and prospects for an integrated gauge theory of gravity and electroweak interactions. Readers are enjoined to reconsider many things they already know from the new perspective of Geometric Calculus!

\section{Historical roots and recent progress}

Geometric Calculus (GC) is an extension of Geometric Algebra (GA) to include differential and integral calculus on manifolds. From a practical point of view, it can be regarded as an extension of tensor algebra and calculus to integrate spinors and differential forms in a unified, coordinate-free mathematical system. GA has its roots in the work of Grassmann and Clifford in the middle of the nineteenth century, but its development as a unified mathematical language for physics did not begin until 1966. ${ }^{1}$ Details of this development are reviewed in [2-4], and a comprehensive treatment is given in [5]. To date, there are more than 10 books and 200 articles on GA and its applications to physics, mathematics, engineering and computer science. 
Most of the GA literature can be traced via websites, ${ }^{6}$ from which all the articles cited in this report can be downloaded.

The specialization of GA to Minkowski spacetime is called Spacetime Algebra (STA). As explained below, STA clarifies the geometric significance of the Dirac Algebra and thereby extends its range of application to the whole of physics. In particular, STA provides the essential mathematical framework for the new Gauge Theory Gravity (GTG).

The foundations of GTG are fully expounded in a seminal paper ${ }^{7}$, so we can concentrate on highlights of its unique features. GTG is a gauge theory on Minkowski spacetime, but locally it is equivalent to General Relativity (GR), so it can be regarded as an alternative formulation of GR. ${ }^{8}$ However, GTG reformulates (or one might say, replaces) Einstein's vague principles of equivalence and general relativity with sharp gauge principles that have clear physical consequences (Section IV). These gauge principles are more than mere rephrasing of Einstein's ideas. They lead to intrinsic mathematical methods that simplify modeling and calculation in GR and clarify physical meaning of terms at every stage. In particular, they provide clean separation between gauge transformations and coordinate transformations, thus resolving a point of longstanding confusion in GR. Moreover, GTG simplifies and clarifies the analysis of singularities, for example, in assignment of time direction to a black hole horizon. Finally, since tensors and spinors are fully integrated in STA, GTG unifies classical GR and relativistic quantum mechanics with a common system of gauge principles. Besides facilitating the application of quantum mechanics to astrophysics, this opens up new possibilities for a grand unification of gravitation and electroweak theories, as explained in Section V.

Claims about the felicity and power of GTG are amply supported by a steady stream of recent publications. These include clarification and simplification of Kerr solutions, ${ }^{11-14}$ new solutions of the Dirac equation in a black hole field, ${ }^{9,10}$ and new results from cosmological modeling with conformal GA. ${ }^{15-18} \mathrm{~A}$ few words are in order to describe what these substantial publications have to offer.

In GTG the curvature tensor for a Kerr black hole with mass M and angular momentum $L$ has the marvelously compact form: ${ }^{4,7}$

$$
R(B)=-\frac{M}{2(r-i L \cos \theta)^{3}}(B+3 \hat{\mathbf{x}} B \hat{\mathbf{x}}),
$$

This expresses the curvature $R(B)$ as a bivector-valued function of a bivector variable (explained below). As the notation suggests, $\hat{\mathbf{x}}$ is the radial unit vector from the origin.

Of course, $R(B)$ reduces to the Schwarzschild curvature when $L=0$. The "complex" denominator is familiar from the literature, but only GTG reveals that the unit imaginary $i$ is properly identified as a pseudoscalar. The simplicity of $R(B)$ belies the subtlety of Kerr geometry and the unsolved problem of matching it to a physically reasonable model of a rotating source. New mathematical techniques to attack that problem for axisymmetric systems are introduced in [9], where, among 
other things, asymptotic uniqueness of the Kerr solution is proven.

A rigorous analysis of singularities in Kerr-Schild geometries is given in [5]. Schwarzschild and Vaidya solutions (without maximal extensions!) are shown to result from a delta-function point source. For the Reissner-Nordstrom solution it is proved that gravity removes the divergent self-energy of classical electromagnetism. Analysis of the Kerr solution at the source reveals a disk of tension surrounded by a matter ring singularity, and physical significance of this structure is discussed.

Turning to the important astrophysical problem of characterizing fermion interactions with black holes: Its relativistic nature calls for an attack using the Dirac equation. However, there is a paucity of such studies, perhaps owing to clumsiness of the standard matrix methods, which have yielded unsatisfactory results in the past. However, the STA version of the Dirac equation is easier to solve than the matrix version, as demonstrated by its use in the studies of black hole quantum mechanics. ${ }^{11-14}$ Ironically, though each of the calculations was first done with STA, some calculations had to be translated into matrix form to just satisfy journal referees. In [13], for example, translation to matrix form took longer than initial solution of the problem using STA.

The gravitational analog of the Mott formula for Coulomb scattering is derived in [11] for the first time. This is extended to next order in the Born series in [14], where numerical calculations for absorption, scattering cross section and polarization are compared with theoretical expectations. In [12] absorption cross sections are calculated for a range of gravitational couplings and initial conditions, and experimental signatures are studied in detail. The gravitational analog of the hydrogen atom spectrum is derived in [13] for the first time. The eigenstates have complex energies. For small couplings, real parts closely follow a hydrogen-like spectrum. Imaginary parts give decay times due to absorption properties of the hole. Deformation of the spectrum as coupling strength increases is thoroughly studied.

On the cosmological front, an extension of STA called conformal GA has been used for a new approach to constructing inflationary models in closed universes. Conformal embedding of closed-universe models in a de Sitter background suggests a quantization condition on the available conformal time that is explored in $[15$, 16]. The result is a predictive model producing reasonable values for the major cosmological parameters and an excellent fit to the most recent CMB radiation data. To fully appreciate the theoretical depth of this work, one needs familiarity with the surprising subtleties of conformal GA, which are nicely expounded in $[17,18]$.

\section{Spacetime Algebra}

For physicists familiar with the Dirac matrix algebra, the quickest approach to STA is by reinterpreting the Dirac matrices as an orthonormal vector basis $\left\{\gamma_{\mu} ; \mu=\right.$ $0,1,2,3\}$ for a $4 \mathrm{D}$ real Minkowski vector space with signature specified by the rules:

$$
\gamma_{0}^{2}=1 \quad \text { and } \quad \gamma_{1}^{2}=\gamma_{2}^{2}=\gamma_{3}^{2}=-1
$$


The frame $\left\{\gamma_{\mu}\right\}$ generates the Spacetime Algebra (STA), an associative geometric algebra that is isomorphic to the Dirac algebra (over the reals - no complex scalars needed!). The product $\gamma_{\mu} \gamma_{\nu}$ of two vectors is called the geometric product. The usual inner product of vectors is defined by

$$
\gamma_{\mu} \cdot \gamma_{\nu} \equiv \frac{1}{2}\left(\gamma_{\mu} \gamma_{\nu}+\gamma_{\nu} \gamma_{\mu}\right)=\eta_{\mu} \delta_{\mu \nu},
$$

where $\eta_{\mu}=\gamma_{\mu}^{2}$ is the signature indicator. The outer product

$$
\gamma_{\mu} \wedge \gamma_{\nu} \equiv \frac{1}{2}\left(\gamma_{\mu} \gamma_{\nu}-\gamma_{\nu} \gamma_{\mu}\right)=-\gamma_{\nu} \wedge \gamma_{\mu}
$$

defines a new entity called a bivector (or 2-vector), which can be interpreted as a directed plane segment representing the plane containing the two vectors.

A complete basis for STA is given by the set:

$\begin{array}{lllll}1 & \left\{\gamma_{\mu}\right\} & \left\{\gamma_{\mu} \wedge \gamma_{\nu}\right\} & \left\{\gamma_{\mu} i\right\} & i \\ 1 \text { scalar } & 4 \text { vectors } & 6 \text { bivectors } & 4 \text { trivectors } & 1 \text { pseudoscalar } \\ \text { grade 0 } & \text { grade 1 } & \text { grade 2 } & \text { grade 3 } & \text { grade 4 }\end{array}$

where the unit pseudoscalar

$$
i \equiv \gamma_{0} \gamma_{1} \gamma_{2} \gamma_{3}
$$

squares to -1 , anticommutes with all odd grade elements and commutes with even grade elements. Thus, STA is a linear space of dimension $1+4+6+4+1=2^{4}=16$.

A generic element in STA is called a multivector. Any multivector can be expressed as a linear combination of the basis elements. For example, a bivector $B$ has the expansion

$$
B=\frac{1}{2} B^{\mu \nu} \gamma_{\mu} \wedge \gamma_{\nu}
$$

with its "scalar components" $B^{\mu \nu}$ in the usual tensorial form. For components, we use the usual tensor algebra conventions for raising and lowering indices and summing over repeated upper and lower index pairs.

Any multivector $M$ can be written in the expanded form

$$
M=\alpha+a+B+b i+\beta i=\sum_{k=0}^{4}\langle M\rangle_{k},
$$

where $\alpha=\langle M\rangle_{0} \equiv\langle M\rangle$ and $\beta$ are scalars, $a=\langle M\rangle_{1}$ and $b$ are vectors, and $B=\langle M\rangle_{2}$ is a bivector, while $b i=\langle M\rangle_{3}$ is a trivector (or pseudovector) and $\beta i=\langle M\rangle_{4}$ is a pseudoscalar.

A multivector is said to be even/odd if it commutes/aniticommutes with the unit pseudoscalar. From eqn. (7) it is obvious that every multivector can be expressed as a sum of even and odd parts. The even multivectors compose a subalgebra of the STA generated by the bivectors $\left\{\boldsymbol{\sigma}_{k} \equiv \gamma_{k} \gamma_{0} ; k=1,2,3\right\}$, so that

$$
\boldsymbol{\sigma}_{1} \boldsymbol{\sigma}_{2} \boldsymbol{\sigma}_{3}=\gamma_{0} \gamma_{1} \gamma_{2} \gamma_{3}=i
$$


Coordinate-free computations are facilitated by various definitions, such as the operation of reversion, which, for any multivector $M$ in the expanded form (7), can be defined by

$$
\tilde{M}=\alpha+a-B-b i+\beta i .
$$

This operation reverses the order in a product of vectors, so for vectors $a, b, c$ we have $(a b c)^{\sim}=c b a$.

An even multivector $L$ subject to the normalization condition

$$
L \tilde{L}=1
$$

is called a rotor. It follows that every rotor can be expressed in the exponential form

$$
L=e^{\frac{1}{2} B},
$$

where $B$ is a bivector. The rotors form a multiplicative group variously called the spacetime rotor group or spin group $\operatorname{Spin}_{+}(1,3), \mathrm{SU}(2 \mathrm{C})$, or the "spin representation" of the Lorentz rotation group.

The concepts of vector and multivector have been defined by algebraic rules specified above. Alternatively, vectors are often defined by their behavior under a transformation group. To keep these two concepts separate, we will use the term tensor to refer to the latter. More generally, we distinguish two kinds of multivectors, tensors and spinors, by the way they transform. Under the rotor Lorentz group $\{L\}$, a tensor $F$ obeys the transformation law

$$
L: F \quad \rightarrow \quad F^{\prime}=L F \tilde{L},
$$

while a spinor $\psi$ obeys

$$
L: \psi \quad \rightarrow \quad \psi^{\prime}=L \psi .
$$

Evidently spinors are more fundamental than tensors, because every spinor $\psi$ determines the tensor transformation law

$$
L: \psi \Gamma \tilde{\psi} \quad \rightarrow \quad \psi^{\prime} \Gamma \tilde{\psi}^{\prime}=L(\psi \Gamma \tilde{\psi}) \tilde{L}
$$

for any fixed multivector $\Gamma$, while one cannot construct spinors from tensors.

\section{Real Quantum Mechanics}

In real QM, the electron wave function $\psi=\psi(x)$ is a spinor field in the real STA. For the electron with charge $e$ and mass $m_{e}$, the field equation for $\psi$ is the real Dirac equation ${ }^{3}$

$$
\gamma^{\mu} \partial_{\mu}\left(\psi i \boldsymbol{\sigma}_{3} \hbar-e A_{\mu} \psi\right)=m_{e} \psi \gamma_{0},
$$

or, in manifestly coordinate-free form,

$$
\partial \psi i \boldsymbol{\sigma}_{3} \hbar-e A \psi=m_{e} \psi \gamma_{0},
$$


where $A=A_{\mu} \gamma^{\mu}$ is the electromagnetic vector potential and the vector derivative $\partial \equiv \gamma^{\mu} \partial_{\mu}$ will be recognized as the famous differential operator introduced by Dirac, except that the $\gamma^{\mu}$ are vectors rather than matrices. This equation is isomorphic to the standard matrix version of the Dirac equation, so it has identical physical content. However, it has the great advantage of representing geometric structure explicitly that is inherent but hidden in the matrix version. In particular, the unit imaginary in the matrix version is explicitly identified with the spacelike bivector $i \boldsymbol{\sigma}_{3}=\gamma_{2} \gamma_{1}$, which indeed does square to -1 .

The real spinor field $\psi=\psi(x)$ in (15) is an even multivector with the invariant form $^{3}$

$$
\psi=\left(\rho e^{i \beta}\right)^{1 / 2} R,
$$

where $R=R(x)$ is a rotor field and $\rho$ and $\beta$ are scalar-valued functions. From (11) it is clear that $R$ is a 6 -parameter function, so $\psi$ has eight degrees of freedom, as required for equivalence with the standard matrix form of the Dirac wave function. This kind of spinor is called an operator spinor, because it operates on fixed vectors to create tensor "observables."

Thus, the Dirac wave function determines a set of local observables

$$
\psi \gamma_{\mu} \tilde{\psi}=\rho e_{\mu}
$$

where $\rho=\rho(x)$ is a scalar probability density and

$$
e_{\mu}=R \gamma_{\mu} \tilde{R}
$$

is a frame field of orthonormal vectors. The vector field $\psi \gamma_{0} \tilde{\psi}=\rho e_{0}$ is the Dirac probability current, which doubles as a charge current when multiplied by the charge $e$. The vector field $e_{3}=R \gamma_{3} \tilde{R}$ specifies the local direction of electron spin. The vector fields $e_{1}$ and $e_{2}$ specify the local phase of the electron, and $e_{2} e_{1}=R \gamma_{2} \gamma_{1} \tilde{R}$ relates the unit imaginary in the Dirac equation to electron spin. Full justification for these interpretations is given elsewhere. ${ }^{3,19}$

We need several other features of standard QM translated into real Dirac theory. Multiplication of the Dirac equation (15) on the right by $\sigma_{2}$ has a net effect of changing the sign of the electric charge. So (suppressing an inconsequential phase factor) we can define a positron wave function $\psi^{C} \equiv \psi \boldsymbol{\sigma}_{2}$ that satisfies the charge conjugate equation

$$
\gamma^{\mu}\left(\partial_{\mu} \psi^{C} i \boldsymbol{\sigma}_{3} \hbar+e A_{\mu} \psi^{C}\right)=m_{e} \psi^{C} \gamma_{0} .
$$

In the matrix theory a chirality operator is defined by $\gamma_{5} \equiv-i^{\prime} \gamma_{0} \gamma_{1} \gamma_{2} \gamma_{3}$ where $i^{\prime}$ is the unit imaginary in the matrix theory. Recalling the definition of the unit pseudoscalar $i$, we translate this into a real operator defined by

$$
\gamma_{5} \psi \equiv i \psi\left(-i \boldsymbol{\sigma}_{3}\right)=\psi \boldsymbol{\sigma}_{3} .
$$

This enables a chiral decomposition of the wave function

$$
\psi=\psi_{l}+\psi_{r}
$$


where left-handed and right-handed chiral eigenstates are defined, respectively by

$$
\begin{gathered}
\psi_{l} \equiv \psi \frac{1}{2}\left(1-\boldsymbol{\sigma}_{3}\right)=-\psi_{l} \boldsymbol{\sigma}_{3}, \\
\psi_{r} \equiv \psi \frac{1}{2}\left(1+\boldsymbol{\sigma}_{3}\right)=\psi_{r} \boldsymbol{\sigma}_{3},
\end{gathered}
$$

The usual Lagrangian for a Dirac electron can be put in the form

$$
\begin{aligned}
\mathcal{L}_{e}= & <\left(\partial \psi i \boldsymbol{\sigma}_{3} \hbar-e A \psi\right) \gamma_{0} \tilde{\psi}-m \psi \tilde{\psi}>=\hbar<-\partial \psi_{l} i \gamma_{0} \tilde{\psi}_{l}+\partial \psi_{r} i \gamma_{0} \tilde{\psi}_{r}> \\
& -<e A\left(\psi_{l} \gamma_{0} \tilde{\psi}_{l}+\psi_{r} \gamma_{0} \tilde{\psi}_{r}\right)+m_{e}\left(\psi_{l} \tilde{\psi}_{r}+\psi_{r} \tilde{\psi}_{l}\right)>,
\end{aligned}
$$

where the angular brackets signify "scalar part." Note that left and right-handed components are coupled only in the mass term. We shall see that this relates to the Higgs mechanism for generating mass.

The electron Lagrangian is invariant under the electromagnetic gauge transformation:

$$
\begin{aligned}
\psi & \rightarrow \psi^{\prime}=\psi e^{i \boldsymbol{\sigma}_{3 \chi}} . \\
e A & \rightarrow e A^{\prime}=e A-\partial \chi .
\end{aligned}
$$

This shows explicitly that the bivector $i \sigma_{3}=\gamma_{2} \gamma_{1}$ is the generator of electromagnetic gauge transformations in the Dirac equation. It follows that gauge transformations are expressions of spacetime geometry in real QM. We shall see that this insight has implications for electroweak gauge theory.

\section{Gauge Theory Gravity with Real Quantum Mechanics}

In Gauge Theory Gravity (GTG) developed by Lasenby, Doran and Gull, ${ }^{7}$ the fundamental geometric entity is the Dirac spinor rather than the line element of GR. This greatly simplifies the integration of gravity theory with quantum mechanics. The Dirac equation (15) generalizes immediately to

$$
g^{\mu}\left(D_{\mu} \psi i \boldsymbol{\sigma}_{3} \hbar-e A_{\mu} \psi\right)=m_{e} \psi \gamma_{0},
$$

where a coordinate frame of vector fields is defined by

$$
g^{\mu}=h_{a}^{\mu} \gamma^{a},
$$

a spinor coderivative $D_{\mu}$ is defined by

$$
D_{\mu} \psi=\left(\partial_{\mu}+\frac{1}{2} \omega_{\mu}\right) \psi
$$

with a bivector-valued "spin connexion" defined by

$$
\omega_{\mu}=\frac{1}{2} \omega_{\mu}^{a b} \gamma_{a} \wedge \gamma_{b}
$$

This will be recognized as formally identical to the usual formulation of the Dirac equation in GR, with the coefficients $h_{a}^{\mu}=g^{\mu} \cdot \gamma_{a}$ identified as components of a vierbein. So what's new besides the fact that $\left\{\gamma^{a}\right\}$ is a frame of constant vectors 
instead of matrices? Only a brief outline of the answer can be given here; full details are given in the references to GTG already cited.

GTG formulates gravity as a gauge field theory on the flat background of Minkowski spacetime. Each spactime point $x$ is a vector that can be parametrized locally by an arbitrary set of coordinates $\left\{x^{\mu}\right\}$. Without loss of generality we can employ rectangular coordinates so that $x=x^{\mu} \gamma_{\mu}$ and $\partial_{\mu} x=\gamma_{\mu}$.

In GTG Einstein's principles of covariance and equivalence are replaced, respectively, by two gauge principles:

Displacement Gauge Invariance: The equations of physics must be invariant under arbitrary smooth remappings of events onto spacetime.

Rotation Gauge Covariance: The equations of physics must be covariant under local Lorentz rotations.

These principles have well-defined mathematical implementations. Displacement invariance requires existence of a differentiable gauge tensor $\bar{h}$ so that

$$
g^{\mu}=\bar{h}\left(\gamma^{\mu}\right)=h_{a}^{\mu} \gamma^{a},
$$

This elevates the vierbein from an auxiliary quantity to a fundamental theoretical entity.

The gauge tensor must be nonsingular, so it has an inverse

$$
g_{\mu}=\underline{h}^{-1}\left(\gamma_{\mu}\right) .
$$

The usual metric tensor is determined by the gauge tensor:

$$
g_{\mu \nu} \equiv g_{\mu} \cdot g_{\nu}=\underline{h}^{-1}\left(\gamma_{\mu}\right) \cdot \underline{h}^{-1}\left(\gamma_{\nu}\right) .
$$

The metric tensor is now an auxiliary quantity.

Rotation gauge covariance is implemented by requiring local covariance of tensors and spinors under the Lorentz rotations (12) and (13) where $L=L(x)$ is an arbitrary position dependent rotor field.

The usual gauge covariant derivative (29) is then defined by by introducing the connexion (30) so that

$$
L: D_{\mu} \psi \quad \rightarrow \quad L\left(D_{\mu} \psi\right)=D_{\mu}^{\prime} \psi^{\prime}=\left(\partial_{\mu}+\frac{1}{2} \omega_{\mu}^{\prime}\right) \psi^{\prime} .
$$

Accordingly, the connexion must obey the familiar gauge theory transformation law

$$
L: \omega_{\mu}=\omega\left(g_{\mu}\right) \quad \rightarrow \quad \omega_{\mu}^{\prime}=L \omega_{\mu} \tilde{L}-2\left(\partial_{\mu} L\right) \tilde{L} .
$$

As the gauge field $g^{\nu}=\bar{h}\left(\gamma^{\nu}\right)$ is a tensor field, its coderivative is given by

$$
D_{\mu} g^{\nu}=\partial_{\mu} g^{\nu}+\omega_{\mu} \times g^{\nu},
$$

where the commutator product is defined by $A \times B \equiv \frac{1}{2}(A B-B A)$.

The curvature tensor, expressed as a bivector-valued linear function of a bivector variable $R(a \wedge b)=a^{\mu} b^{\nu} R\left(g_{\mu} \wedge g_{\nu}\right)$, is obtained immediately from the commutator of coderivatives. Thus,

$$
\left[D_{\mu}, D_{\nu}\right] \psi=\frac{1}{2} R\left(g_{\mu} \wedge g_{\nu}\right) \psi,
$$


where

$$
R\left(g_{\mu} \wedge g_{\nu}\right)=\partial_{\mu} \omega_{\nu}-\partial_{\nu} \omega_{\mu}+\omega_{\mu} \times \omega_{\nu} .
$$

This is the fundamental form for curvature in GTG. It follows that the curvature bivector has the gauge covariance property

$$
L: R(a \wedge b) \quad \rightarrow \quad R^{\prime}\left(a^{\prime} \wedge b^{\prime}\right)=L R(L(a \wedge b) \tilde{L}) \tilde{L} .
$$

Gravitational field equations can be obtained from a Lagrangian. ${ }^{7}$ As GTG postulates two basic variables, the gauge field and the connexion, there must be two field equations. The first is equivalent to Cartan's "first fundamental form:"

$$
D \wedge g^{\nu} \equiv g^{\mu} \wedge D_{\mu} g^{\nu}=\theta^{\nu},
$$

where the torsion $\theta^{\nu}=\theta\left(g^{\nu}\right)$ is a linear bivector-valued function of a vector field. In GR the torsion vanishes, in which case the connexion is Riemannian, and (40) can be solved algebraically to give the connexion as a function of the gauge tensor and its first derivatives. However, it has been shown ${ }^{7}$ that a matter field described by the Dirac equation gives rise to a non-vanishing torsion.

The other field equation is, of course, Einstein's equation:

$$
G\left(g^{\mu}\right)=\kappa T\left(g^{\mu}\right),
$$

where components of the matter energy-momentum tensor are given by $T^{\nu \mu}=$ $g^{\nu} \cdot T\left(g^{\nu}\right)$. For Einstein's tensor, STA gives us the elegant compact form

$$
G\left(g^{\mu}\right)=\frac{1}{2}\left(g^{\mu} \wedge g^{\alpha} \wedge g^{\beta}\right) \cdot R\left(g_{\alpha} \wedge g_{\beta}\right) .
$$

\section{Gravelectroweak Gauge Theory: Standard Model}

The gravelectric Dirac equation (27) is covariant under the gravelectric gauge transformation:

$$
\psi \quad \rightarrow \quad \psi^{\prime}=L \psi U,
$$

where $L$ is a Lorentz rotor and $U=e^{i \boldsymbol{\sigma}_{3} \chi}$. This can be regarded as a spacetime transformation acting on the left of $\psi$ and an (internal) isospace transformation acting on the right.

According to standard electroweak theory, the electromagnetic gauge group is only one part of the electroweak group. But real Dirac theory tells us that the electromagnetic gauge generator is a geometric object $i \sigma_{3}$ acting on the right of $\psi$, so we should expect similar geometric objects generating the rest of the electroweak group. Indeed, the most general gauge transformation covariant on the right side of (27) satisfies

$$
\tilde{U} \gamma_{0} U=\gamma_{0}
$$

The general solution of this equation has the form

$$
U=e^{\frac{1}{2} i \boldsymbol{\theta}} e^{\frac{1}{2} i \chi},
$$


where $\boldsymbol{\theta}=\theta_{1} \boldsymbol{\sigma}_{1}+\theta_{2} \boldsymbol{\sigma}_{2}+\theta_{3} \boldsymbol{\sigma}_{3}$. This is exactly the gauge group $\mathrm{SU}(2) \otimes \mathrm{U}(1)$ of electroweak theory. It strongly suggests that the geometric structure of electroweak theory is already inherent in Dirac theory!

Thus we arrive at the question: How can we revise the Dirac equation (27) to incorporate electroweak interactions acting on the right side of the wave function? We shall consider two answers. In this section we see how the structure of the standard model can be incorporated in a way that links it to spacetime geometry with new physical features. In a later section we formulate a simpler alternative to the standard model that certainly will have new physical predictions.

According to the standard model electroweak interactions couple differently to the two chiral eigenstates of the electron. For that purpose, a slick way to separate chiral eigenstates is to multiply the electron wave function $\psi$ by the idempotent (a projection operator) $\frac{1}{2}\left(1+\gamma_{0}\right)\left(1-\boldsymbol{\sigma}_{3}\right)$ to get

$$
\Psi=\psi \frac{1}{2}\left(1+\gamma_{0}\right)\left(1-\boldsymbol{\sigma}_{3}\right)=\psi_{l}+\psi_{r} \gamma_{0} .
$$

This type of spinor is called an ideal spinor, because its values are elements of a minimal left ideal in the STA. Note that the chiral components of the operator spinor (22) have been neatly separated into even and odd parts of the ideal spinor (46).

Multiplying (27) by the same idempotent, we get the Dirac equation in the "ideal form"

$$
-g^{\mu}\left(D_{\mu} \Psi i \hbar+e A_{\mu} \Psi\right)=m_{e} \Psi .
$$

This looks more like the matrix Dirac equation, though with the pseudoscalar $i$ playing the role of unit imaginary. It is, in fact, the first version of the Dirac equation forumlated in the real STA. ${ }^{1}$ It was noted at the time that minimal ideals can be used to incorporate isospace degrees of freedom. However, physical motivation for implementing this possibility had to wait for development of electroweak theory. The key insight was noticing the separation of chiral eigenstates in (46). That paved the way for a leptonic version of Dirac's equation that incorporates all the electroweak interactions. ${ }^{20}$

For the purpose of electroweak theory, we affix a subscript to the electron wave function

$$
\Psi_{e}=\psi_{e} \frac{1}{2}\left(1+\gamma_{0}\right)\left(1-\boldsymbol{\sigma}_{3}\right)=\psi_{l}+\psi_{r} \gamma_{0}=-\Psi_{e} \boldsymbol{\sigma}_{3},
$$

and we define a neutrino wave function ${ }^{20,21}$

$$
\Psi_{\nu}=\psi_{\nu} \frac{1}{2}\left(1-\sigma_{3}\right) \sigma_{1}=\psi_{\nu} \frac{1}{2} \sigma_{1}\left(1+\sigma_{3}\right)=\Psi_{\nu} \sigma_{3} .
$$

The right side of these equations shows that the $\Psi_{e}$ and $\Psi_{\nu}$ are different eigenstates of $\boldsymbol{\sigma}_{3}$ operating from the right. In other words, they are elements of independent minimal left ideals. The neutrino wave function in (49) is left-handed, with no righthanded (or odd) component. In common parlance, it represents a 2-component Weyl neutrino. 
Now we regard electron and neutrino as different states of a single lepton with wave function

$$
\Psi=\Psi_{e}+\Psi_{\nu}=\Psi_{l}+\Psi_{r} .
$$

The left-handed and right-handed components are defined, respectively, by

$$
\begin{gathered}
\Psi_{l} \equiv \frac{1}{2}(\Psi-i \Psi i)=\psi_{e} \frac{1}{2}\left(1-\sigma_{3}\right)+\psi_{\nu} \frac{1}{2}\left(1-\sigma_{3}\right) \sigma_{1}, \\
\Psi_{r} \equiv \frac{1}{2}(\Psi+i \Psi i)=\psi_{e} \frac{1}{2}\left(1+\sigma_{3}\right) \gamma_{0} .
\end{gathered}
$$

Following the standard model, we assume a gravelectroweak gauge transformation of the form

$$
\Psi \quad \rightarrow \quad \Psi^{\prime}=L\left(\Psi_{l} U+\Psi_{r} \tilde{U} U\right),
$$

where $U$ is defined as before in (45), so $\tilde{U} U=e^{i \chi}$. One says that $\Psi_{l}$ transforms as an isospinor (on the right), while $\Psi_{r}$ transforms as an isoscalar.

Now we can define a generalized coderivative in the usual way:

$$
\mathcal{D}_{\mu} \Psi=D_{\mu} \Psi-\Psi_{l} i W_{\mu}-\Psi_{r} i g^{\prime} B_{\mu},
$$

with $\hbar=1$ from here on. In perfect analogy to the gravitational connexion (35) the nonabelian electroweak connexion obeys the transformation law

$$
W_{\mu} \quad \rightarrow \quad W_{\mu}^{\prime}=\tilde{U} W_{\mu} U-\tilde{U} \partial_{\mu} U
$$

Using notation of the standard model as closely as possible, the connexion has the form

$$
\begin{aligned}
2 W_{\mu} & \equiv g \mathbf{A}_{\mu}-g^{\prime} B_{\mu} \\
& =\frac{g}{\sqrt{2}}\left[W_{\mu}^{+}\left(1+\boldsymbol{\sigma}_{3}\right) \boldsymbol{\sigma}_{1}+W_{\mu}^{-}\left(1-\boldsymbol{\sigma}_{3}\right) \boldsymbol{\sigma}_{1}\right]+g A_{\mu}^{3} \boldsymbol{\sigma}_{3}-g^{\prime} B_{\mu},
\end{aligned}
$$

where

$$
W_{\mu}^{ \pm} \equiv \frac{1}{\sqrt{2}}\left(A_{\mu}^{1} \mp i A_{\mu}^{2}\right) .
$$

Introducing the Weinberg angle in the standard way, we have

$$
\begin{gathered}
g A_{\mu}^{3} \boldsymbol{\sigma}_{3}-g^{\prime} B_{\mu}=-e A_{\mu}\left(1-\boldsymbol{\sigma}_{3}\right)-\frac{g}{\cos \theta_{W}} Z_{\mu}\left(\boldsymbol{\sigma}_{3} \cos ^{2} \theta_{W}+\sin ^{2} \theta_{W}\right), \\
g^{\prime} B_{\mu}=e A_{\mu}+\frac{g}{\cos \theta_{W}} Z_{\mu} \sin ^{2} \theta_{W},
\end{gathered}
$$

where $e=g \sin \theta_{W}=g^{\prime} \cos \theta_{W}$ relates the electron charge to the weak coupling constants $g$ and $g^{\prime}$.

It is convenient to put all this together in a single lepton Lagrangian:

$$
\begin{aligned}
h \mathcal{L}_{\text {lep }} & =<\mathcal{D} \Psi i \gamma_{0} \tilde{\Psi}>\equiv<g^{\mu} \mathcal{D}_{\mu} \Psi i \gamma_{0} \tilde{\Psi}> \\
& =<D \Psi i \gamma_{0} \tilde{\Psi}>+<g^{\mu}\left(\Psi_{l} W_{\mu}+\Psi_{r} g^{\prime} B_{\mu}\right) \gamma_{0} \tilde{\Psi}> \\
& =<D \Psi i \gamma_{0} \tilde{\Psi}>+\frac{g}{2 \sqrt{2}}\left(W^{+} J_{-}+W^{-} J_{+}\right)-e A \cdot J+\frac{g}{\sqrt{2}} Z \cdot J_{Z} .
\end{aligned}
$$


The sundry quantities in this Lagrangian are defined in the following. The scale factor $h \equiv \operatorname{det} \underline{h}$ is necessary to make $\mathcal{L}_{\text {lep }}$ a gauge invariant Langrangian density.

The kinetic-gravitation component of the Lagrangian has the familiar form

$$
<D \Psi i \gamma_{0} \tilde{\Psi}>=<g^{\mu}\left(\partial_{\mu}+\frac{1}{2} \omega_{\mu}\right) \Psi i \gamma_{0} \tilde{\Psi}>,
$$

which we will not explicate further, as we are most interested in the electroweak interactions.

As usual, the electromagnetic vector potential is $A=g^{\mu} A_{\mu}$. The weak gauge fields are the charged fields $W^{+}=g^{\mu} W_{\mu}^{+}=\tilde{W}^{-}$(having vector and pseudovector parts) and the neutral vector field $Z=g^{\mu} Z_{\mu}$. After some fairly easy computations (exploiting the fact that odd terms cannot have a scalar part), we arrive at the following explicit forms for the corresponding lepton currents. The usual Dirac current is given by

$$
J=\psi_{e} \gamma_{0} \tilde{\psi}_{e}=\psi_{e} \frac{1}{2}\left(\gamma_{0}-\gamma_{3}\right) \tilde{\psi}_{e}+\psi_{e} \frac{1}{2}\left(\gamma_{0}+\gamma_{3}\right) \tilde{\psi}_{e},
$$

where the right side separates the contributions of left-and right-handed components. The charged and neutral weak currents are

$$
\begin{gathered}
J_{-}=\psi_{e}\left(\gamma_{0}-\gamma_{3}\right) \tilde{\psi}_{\nu}=\tilde{J}_{+} \\
J_{Z}=\psi_{\nu}\left(\gamma_{0}-\gamma_{3}\right) \tilde{\psi}_{\nu}-\psi_{e} \frac{1}{2}\left(\gamma_{0}-\gamma_{3}-4 \sin ^{2} \theta_{W} \gamma_{0}\right) \tilde{\psi}_{e} .
\end{gathered}
$$

This completes our characterization of the gravelectroweak Lagrangian (60) for leptons.

The rest of the standard model is now easily transcribed into STA. Lagrangians for the gauge fields carry over with no essential change. The Higgs mechanism has been transcribed by Antony Lewis in unpublished notes. ${ }^{22}$

The electron mass can be incorporated in the Lagrangian (60) by subtracting the term

$$
<\Psi \underline{\Psi} \tilde{\Psi}>=m_{e}<\psi_{e} \tilde{\psi}_{e}>,
$$

Higgs theory replaces the mass operator $\underline{m}=m_{e} \gamma_{0}$ by a Higgs field. As shown explicitly in (25), the mass (hence the Higgs mechanism) requires coupling between left- and right-handed electron states. Therefore, if a small neutrino mass is to arise from the same mechanism, a right-handed component must be added to the neutrino wave function (49). In that case, the mass operator would generalize to

$$
\underline{m}=\left[m_{e} \frac{1}{2}\left(1-\boldsymbol{\sigma}_{3}\right)+m_{\nu} \frac{1}{2}\left(1+\boldsymbol{\sigma}_{3}\right)\right] \gamma_{0},
$$

where $m_{\nu}$ is the neutrino mass.

Looking back, we see that Dirac theory does indeed have degrees of freedom sufficient to incorporate electroweak theory in a fairly straightforward way. One thing new stands out: the idempotent $\frac{1}{2}\left(1-\sigma_{3}\right)$ that breaks the symmetry of 
the electroweak connexion in (56) by separating electromagnetic from weak interactions, also separates left and right-handed lepton states in (51) and (52). In the standard model this relation between symmetry breaking and chiral eigenstates is incorporated as an independent assumption. In this respect, therefore, electroweak theory is simplified by its geometrization in STA. Though this may not produce new physical predictions, it does reveal new possibilities for modifying electroweak theory that surely will produce new predictions. One such possibility is developed next.

\section{Zitterbewegung in Dirac Theory}

Having aligned the standard model with geometry of the Dirac equation, we notice that one prominent feature of Dirac theory is missing, namely, the zitterbewegung (zbw) of the electron. The zbw was discovered and given its name by Schroedinger in an analysis of free particle solutions of the Dirac equation. ${ }^{23}$ It has since been recognized as a general feature of electron phase fluctuations and proposed as a fundamental principle of QM. ${ }^{19,24}$

One reason that the significance of zbw has been consistently overlooked, especially in electroweak theory, is that the relevant observables are not among the so-called "bilinear covariants," from which observable currents are constructed in the standard model. I refer to the vector fields $\psi \gamma_{1} \tilde{\psi}=\rho e_{1}$ and $\psi \gamma_{2} \tilde{\psi}=\rho e_{2}$, identified as observables in (18). The standard model deals only with $\psi \gamma_{0} \tilde{\psi}=\rho e_{0}$ and $\psi \gamma_{3} \tilde{\psi}=\rho e_{3}$, especially in combination to form chiral currents in (62), (63), and (64).

One way to recognize the significance of the observables $e_{2}$ and $e_{1}$ is to note that they rotate with twice the electron phase along streamlines of the conserved Dirac current. The rotation rate for a free electron is the $2 m_{e} / \hbar \approx 1 \mathrm{ZHz}$ (zettaHertz), the $z b w$ frequency found by Schroedinger. This rate varies in the presence of interactions but still remains outside the range of direct observation.

Other features of the zbw may be detectible, however. In particular, it has often been suggested that the electron's magnetic moment is generated by a circulating charged current. That suggestion is elevated to a principle by replacing the charged Dirac current $e \psi \gamma_{0} \tilde{\psi}$ by the $z b w$ current $e \psi\left(\gamma_{0}-\gamma_{2}\right) \tilde{\psi}=e \rho\left(e_{0}-e_{2}\right)$. Obviously, the zbw current is analogous to the left-handed chiral current $\psi_{e} \frac{1}{2}\left(\gamma_{0}-\gamma_{3}\right) \tilde{\psi}_{e}$ with $\gamma_{3}$ replaced by $\gamma_{2}$. Equation (62) expresses the Dirac current as a sum of left- and right-handed chiral currents. Therefore, we can incorporate zbw into electroweak theory by dropping the right-handed current and replacing the left-handed current by the zbw current.

There is no need for a zbw analog of the right-handed chiral current. Looking over the standard model in the preceding section, it is evident that the right-handed current plays only a minor role. Its main function is to balance the left-handed current to produce the Dirac current, as shown in (62). The theory can be simplified considerably once that function is seen to be unnecessary. We see how in the next 
section.

\section{Gravelectroweak Gauge Theory: Majorana Model}

In Section [6] we saw how the standard model can be incorporated into real Dirac theory using the chiral projection operator $\frac{1}{2}\left(1-\sigma_{3}\right)$. By a completely analogous procedure we now construct an alternative model using the Majorana projection operator $\frac{1}{2}\left(1-\sigma_{2}\right)$, so named for reasons that are obvious below.

As in Section 4, we begin with a real Dirac wave function $\Psi$, but here we identify it as a lepton wave function with electron and neutrino components defined as follows:

$$
\Psi=\Psi_{e}+\Psi_{\nu}=\psi_{e} \frac{1}{2}\left(1-\sigma_{2}\right)+\psi_{\nu} \frac{1}{2}\left(1-\sigma_{2}\right) \sigma_{3},
$$

with

$$
\begin{gathered}
\Psi_{e}=\frac{1}{2}\left(\Psi-\Psi \sigma_{2}\right)=\psi_{e} \frac{1}{2}\left(1-\sigma_{2}\right)=-\Psi_{e} \sigma_{2}, \\
\Psi_{\nu}=\frac{1}{2}\left(\Psi+\Psi \sigma_{2}\right)=\psi_{\nu} \frac{1}{2}\left(1-\sigma_{2}\right) \sigma_{3}=\Psi_{\nu} \sigma_{2} .
\end{gathered}
$$

Note the analogy with equations (48) and (49). Here $\Psi_{e}$ and $\Psi_{\nu}$ are eigenstates of $\boldsymbol{\sigma}_{2}$ operating on the right, which, as is evident from (20), makes them eigenfunctions of the charge conjugation operator, commonly called Majorana states in the literature.

Note that we have introduced a major shift in physical interpretation of the Dirac wave function, decomposing it into charged and neutral components rather than the usual decomposition into positive and negative energy components. This shift is not so radical as it might seem at first, because it is completely analogous to the decomposition of (51) in the standard model. Of course, we have to modify interactions in the Dirac equation to be consistent with the new interpretation, but those modifications are the same as in the standard model.

With the change in physical interpretation of the Dirac wave function comes a change in physical interpretation of operators. In particular, the antiparticle conjugate of the lepton wave function is

$$
\Phi \equiv \Psi \sigma_{3}=\varphi_{\bar{e}} \frac{1}{2}\left(1-\sigma_{2}\right)+\varphi_{\bar{\nu}} \frac{1}{2}\left(1-\sigma_{2}\right) \sigma_{3} .
$$

As in the standard model, we assume a gravelectroweak gauge transformation of the form

$$
\Psi \quad \rightarrow \quad \Psi^{\prime}=L \Psi U
$$

As before, the generalized coderivative for this gauge group has the form

$$
\mathcal{D}_{\mu} \Psi=D_{\mu} \Psi-\Psi i W_{\mu},
$$


where $D_{\mu}=\partial_{\mu}+\frac{1}{2} \omega_{\mu}$ and the electroweak connexion is given by

$$
\begin{aligned}
2 W_{\mu} \equiv & g \mathbf{A}_{\mu}-g^{\prime} B_{\mu}=\frac{g}{\sqrt{2}}\left[W_{\mu}^{+}\left(1+\boldsymbol{\sigma}_{2}\right) \boldsymbol{\sigma}_{3}+W_{\mu}^{-}\left(1-\boldsymbol{\sigma}_{2}\right) \boldsymbol{\sigma}_{3}\right] \\
& -e A_{\mu} \frac{1}{2}\left(1-\boldsymbol{\sigma}_{2}\right)-\frac{g}{\cos \theta_{W}} Z_{\mu}\left(\boldsymbol{\sigma}_{2} \cos ^{2} \theta_{W}+\sin ^{2} \theta_{W}\right),
\end{aligned}
$$

where

$$
W_{\mu}^{ \pm} \equiv \frac{1}{\sqrt{2}}\left(A_{\mu}^{3} \mp i A_{\mu}^{1}\right) .
$$

This differs from the standard model only in a permutation of indices due to splitting the connexion with a Majorana projection instead of a chiral projection. All variables and parameters are the same as in the standard model.

A modified Dirac equation for the Majorana model can now be written down immediately:

$$
g^{\mu} \mathcal{D}_{\mu} \Psi i \gamma_{3}=\Psi \underline{m},
$$

where lepton masses have been incorporated in a mass operator

$$
\underline{m}=m_{e} \frac{1}{2}\left(1-\boldsymbol{\sigma}_{2}\right)+m_{\nu} \frac{1}{2}\left(1+\boldsymbol{\sigma}_{2}\right) .
$$

Of course, the mass operator can be replaced by a Higgs mechanism, but we leave that as an open question. There are good reasons to believe that gravity plays a role in determining particle masses. ${ }^{25}$ Unified gravelectroweak theory should help in investigating that possibility.

In constructing a Lagrangian to give us the field equation (75), we note a problem: The mass term in the usual Dirac Lagrangian (25) requires a 4-component wave function, whereas the electron wave function (68) here has only 2 components. A solution is suggested by noting that the usual mass term in (25) couples positive and negative energy states, so the analog here is coupling lepton and antilepton states. Accordingly, we use the antilepton wave function (70) to construct a Lagrangian of the form

$$
h \mathcal{L}_{M a j}=<g^{\mu} \mathcal{D}_{\mu} \Psi i \gamma_{3} \tilde{\Phi}>-<\Psi \underline{m} \tilde{\Phi}>,
$$

Alternatively, we can use $\tilde{\Phi}=-\sigma_{3} \tilde{\Psi}$ to write the Lagrangian as

$$
\begin{aligned}
h \mathcal{L}_{\text {Maj }} & =<g^{\mu} \mathcal{D}_{\mu} \Psi i \gamma_{0} \tilde{\Psi}>+<\Psi \underline{m} \boldsymbol{\sigma}_{3} \tilde{\Psi}> \\
& =<D \Psi i \gamma_{0} \tilde{\Psi}>+<g^{\mu} \Psi W_{\mu} \gamma_{0} \tilde{\Psi}>+<\Psi \underline{m} \boldsymbol{\sigma}_{3} \tilde{\Psi}>.
\end{aligned}
$$

The last line singles out the electroweak interactions for further discussion. It is interesting to note that

$$
2 W_{\mu} \gamma_{0}=g \mathbf{A}_{\mu} \gamma_{0}-g^{\prime} B_{\mu} \gamma_{0}=g A_{\mu}^{k} \gamma_{k}-g^{\prime} B_{\mu} \gamma_{0}
$$

is a spacetime vector, with 4 gauge fields coresponding to 3 spacelike components and a distinguished timelike component. 
The electroweak term in (78) has exactly the same form as in the standard model (60), namely,

$$
<g^{\mu} \Psi W_{\mu} \gamma_{0} \tilde{\Psi}>=\frac{g}{2 \sqrt{2}}\left(W^{+} J_{-}+W^{-} J_{+}\right)-e A \cdot J+\frac{g}{\sqrt{2}} Z \cdot J_{Z} .
$$

The gauge fields $W^{ \pm}, A, Z$ are the same as before, but the lepton currents are somewhat different: The Dirac current is replaced by the $z b w$ current

$$
J=\psi_{e} \frac{1}{2}\left(\gamma_{0}-\gamma_{2}\right) \tilde{\psi}_{e}
$$

The charged and neutral weak currents are

$$
\begin{gathered}
J_{-}=\psi_{e}\left(\gamma_{0}-\gamma_{2}\right) \tilde{\psi}_{\nu}=\tilde{J}_{+} \\
J_{Z}=\psi_{\nu}\left(\gamma_{0}-\gamma_{2}\right) \tilde{\psi}_{\nu}-\psi_{e} \frac{1}{2}\left(\gamma_{0}-\gamma_{2}-4 \sin ^{2} \theta_{W} \gamma_{0}\right) \tilde{\psi}_{e} .
\end{gathered}
$$

This completes our characterization of the Majorana Lagrangian (78) for leptonic gravelectroweak interactions.

So far we have ignored the derivation of field equations from the Lagrangian density, but that involves a subtle point that is often overlooked. Though the point has been made before, ${ }^{7}$ it is worth reviewing to complete the present theory. Regarding $\Psi$ and $\tilde{\Psi}$ as independent variables in the Lagrangian (78) appears to give the field equation (75) immediately when $\tilde{\Psi}$ is varied. But the correct procedure, that regards them as the same variable, gives a different result. To make the point it suffices to consider variation of the kinetic-gravitation part of the Lagrangian, which, conveniently, has the same form (61) in both Majorana and Standard models.

With help of the properties $\langle A\rangle=\langle\tilde{A}\rangle$ and $\langle A B\rangle=\langle B A\rangle$, we obtain

$$
\begin{aligned}
\delta<h^{-1} g^{\mu} \partial_{\mu} \Psi i \gamma_{0} \tilde{\Psi}>= & 2<h^{-1} g^{\mu} \partial_{\mu} \Psi i \gamma_{0} \delta \tilde{\Psi}>+<\partial_{\mu}\left(h^{-1} g^{\mu}\right) \Psi i \gamma_{0} \delta \tilde{\Psi}> \\
& -\partial_{\mu}<h^{-1} g^{\mu} \Psi i \gamma_{0} \delta \tilde{\Psi}>
\end{aligned}
$$

and

$$
\delta<g^{\mu} \frac{1}{2} \omega_{\mu} \Psi i \gamma_{0} \tilde{\Psi}>=\frac{1}{2}<\left(g^{\mu} \omega_{\mu}+\omega_{\mu} g^{\mu}\right) \Psi i \gamma_{0} \delta \tilde{\Psi}>.
$$

These combine to give us

$$
\delta<h^{-1} D \Psi i \gamma_{0} \tilde{\Psi}>=2 h^{-1}<\left[g^{\mu}\left(\partial_{\mu}+\frac{1}{2} \omega_{\mu}\right)+\frac{1}{2} h D_{\mu}\left(h^{-1} g^{\mu}\right)\right] \Psi i \gamma_{0} \delta \tilde{\Psi}>
$$

where

$$
h D_{\mu}\left(h^{-1} g^{\mu}\right)=h \partial_{\mu}\left(h^{-1} g^{\mu}\right)+\omega_{\mu} \times g^{\mu},
$$

and we have dropped the boundary term with vanishing variation.

Completing the variation of other terms we find that the generalized Dirac equation (75) must be amended to the form

$$
g^{\mu} \mathcal{D}_{\mu} \Psi i \gamma_{3}+\frac{1}{2} h D_{\mu}\left(h^{-1} g^{\mu}\right) \Psi i \gamma_{3}=\Psi \underline{m},
$$


The additional term is proportional to the torsion, so it vanishes when the torsion vanishes, but we have noted that is not generally the case.

In the usual way, the commutator of the generalized coderivative (72) gives us a generalized curvature:

$$
\left[\mathcal{D}_{\mu}, \mathcal{D}_{\nu}\right] \Psi=\frac{1}{2} R\left(g_{\mu} \wedge g_{\nu}\right) \Psi+\Psi W_{\mu \nu},
$$

where $R\left(g_{\mu} \wedge g_{\nu}\right)$ is the gravitational curvature (38), and the electroweak curvature has the standard form

$$
\begin{aligned}
W_{\mu \nu} & =i\left(\partial_{\mu} W_{\nu}-\partial_{\nu} W_{\mu}\right)-2 W_{\mu} \times W_{\nu} \\
& =\frac{g}{2} i\left(-\partial_{\mu} \mathbf{A}_{\nu}+\partial_{\nu} \mathbf{A}_{\mu}+2 i \mathbf{A}_{\mu} \times \mathbf{A}_{\nu}\right)+\frac{g^{\prime}}{2} i\left(\partial_{\mu} B_{\nu}-\partial_{\nu} B_{\mu}\right) .
\end{aligned}
$$

Gravelectroweak theory obviously enhances the symmetry between these two curvatures, and thus raises the question as to how far the symmetry should extend throughout the theory. Many people have noted the dyssymmetry in standard gauge field Lagrangians, which are linear in the gravitational curvature but quadratic in the electroweak curvature. Without trying to resolve the issue here, it is worth noting that GA has been used in two studies of Lagrangians that are quadratic in the gravitational curvature. ${ }^{26,27}$

\section{Conclusions}

We have seen how STA enables integration of gravitational and electroweak interactions into a unified gravelectroweak gauge theory with real spinor fields representing basic physical entities. Besides simplifying physical analysis and computations, as documented in the references, this provides a new framework for studying interactions among gauge fields.

The most striking revelation of the new theory is that the real Dirac equation has inherent geometric structure that perfectly matches the structure of electroweak isospace, including precisely the right number of degrees of freedom. This means that electroweak theory can be construed as a theory of spacetime geometry of real spinor fields.

Incorporating the standard Weinberg-Salam model into this geometric framework reveals a natural way to identify the chiral structure of the model with splitting of the gauge fields into charged and neutral components. Though this insight cannot be expected to yield new experimental predictions, it does open new possibilities for improving the theory. The most promising possibility has been dubbed the Majorana model.

We arrived at the Majorana model as a variant of the Standard model simply by a change in projection operator. Chiral splitting of the Dirac wave function by the operator $\frac{1}{2}\left(1-\sigma_{3}\right)$ is motivated experimentally but not theoretically. Majorana splitting by the operator $\frac{1}{2}\left(1-\boldsymbol{\sigma}_{2}\right)$ is motivated theoretically but remains to be tested experimentally. 
Since the models are so similar structurally, it is not immediately clear whether the Majorana model is inconsistent with any current experimental evidence supporting the Standard model. But it is clear that experimental tests can be devised to discriminate between the models.

The crucial test of the Majorana model will be experimental validation of zitterbewegung structure in the electron charge current. There are many possibilities for experimental tests that cannot be addressed here. There are many metaphorical remarks about zitterbewegung in the literature on relativistic QM. It is high time to get serious about zitterbewegung as a real physical phenomenon.

There are other possibilities for experimental tests of the Majorana model, for example, to distinguish Chiral and Majorana states in neutrino mixing.

Of course, if gravelectroweak theory works for leptons it probably works for quarks as well. It remains to be seen if a comparable geometric theory can be devised for strong interactions.

\section{References}

1. D. Hestenes, Space-Time Algebra, (Gordon \& Breach, New York, 1966).

2. D. Hestenes, "Oersted Medal Lecture 2002: Reforming the mathematical language of physics," Am. J. Phys. 71: 104-121 (2003).

3. D. Hestenes, "Spacetime physics with Geometric Algebra," Am. J. Phys. 71: 691-704 (2003).

4. D. Hestenes, "Gauge Theory Gravity with Geometric Calculus," Foundations of Physics 36: 903-970 (2005).

5. C. Doran \& A. Lasenby, Geometric Algebra for Physicists (Cambridge University Press, Cambridge, 2003).

6. GA websites: <modelingnts.la.asu.edu $>$, <www.mrao.cam.ac.uk $>$

7. A. Lasenby, C. Doran \& S. Gull, "Gravity, gauge theories and geometric algebra," Phil. Trans. R. Soc. Lond. A 356: 487-582 (1998).

8. GTG can be regarded as a modernized version of ECKS or $U_{4}$ spin-torsion theory. F. Heyl, P. von der Heyde, D. Kerlick, \& J. Nester, "General relativity with spin and torsion: Foundations and Prospects," Rev. Mod. Phys. 48: 393-416 (1976).

9. C. Doran \& A. Lasenby, "New Techniques for Analyzing Axisymmetric Gravitational Systems. 1. Vacuum Fields," Class. Quant. Grav. 20: 1977-1102 (2003).

10. C. Doran \& A. Lasenby, "Integral Equations, Kerr-Schild fields and gravitational sources," (arXiv:gr-qc/0404081).

11. C. Doran \& A. Lasenby, "Perturbation Theory Calculation of the Black Hole Elastic Scattering Cross Section," it Phys. Rev. D66: (2002) 024006.

12. C. Doran, A. Lasenby, S. Dolan \& I. Hinder, "Fermion absorption cross section of a Schwarzschild black hole," Phys. Rev. D71: 124020 (2005).

13. A. Lasenby, C. Doran, J. Pritchard \& A Caceres, "Bound states and decay times of fermions in a Schwarzschild black hole background," Phys. Rev. D72: 105014 (2005).

14. S. Dolan, C. Doran \& A. Lasenby, "Fermion Scattering by a Schwarzschild black hole," (Submitted to Physical Review).

15. A. Lasenby \& C. Doran, "Conformal models of de Sitter space, initial conditions for inflation and the CMB," To appear in proceeding 'Phi in the Sky,' (Porto, 2004).

16. A. Lasenby \& C. Doran, "Closed Universes, de Sitter Space and Inflation," Phys. Rev. D71: 063502 (2005). 
17. A. Lasenby, "Conformal Geometry and the Universe," To appear in Phil. Trans. R. Soc. Lond. A.

18. A. Lasenby, "Recent Applications of Conformal Geometry," In H. Li, P. Olver and G. Sommer (Eds): IWMM 2004; Lecture Notes in Computer Science 3519 (SpringerVerlag, 2005).

19. D. Hestenes, "Mysteries and Insights of Dirac Theory," Annales de la Fondation Louis de Broglie 28: 390-408 (2003).

20. D. Hestenes, "Space-Time Structure of Weak and Electromagnetic Interactions," Found. Phys. 12: 153-168 (1982).

21. C. Doran \& A. Lasenby, Geometric Algebra for Physicists (Cambridge University Press, Cambridge, 2003). p. 464.

22. A. Lewis, "Electroweak theory," http://cosmologist.info/notes/

23. E. Schroedinger, Sitzungb. Preuss. Akad. Wiss. Phys.-Math. Kl. 24, 418 (1930).

24. D. Hestenes, "The Zitterbewegung Interpretation of Quantum Mechanics," Found. Phys. bf 20: 1213-1232 (1990).

25. K. Rosquist, "Gravitationally induced electromagnetism at the Compton scale," (arXiv:gr-qc/0412064 v2)

26. A. Lewis, C. Doran \& A. Lasenby, "Quadratic Lagrangians and Topology in Gauge Theory Gravity," (arXiv:gr-gc/9910039).

27. A. Lasenby, C. Doran \& R. Heineke, "Analytic solutions to Riemann-squared gravity with background isotropic torsion," (arXiv:gr-gc/0509014). 